\title{
Complete genome sequence of Paludibacter propionicigenes type strain (WB4 ${ }^{\top}$ )
}

\author{
Sabine Gronow ${ }^{1}$, Christine Munk ${ }^{2,3}$, Alla Lapidus ${ }^{2}$, Matt Nolan², Susan Lucas ${ }^{2}$, Nancy \\ Hammon $^{2}$, Shweta Deshpande ${ }^{2}$, Jan-Fang Cheng ${ }^{2}$, Roxane Tapia ${ }^{2,3}$, Cliff Han $^{2,3}$, Lynne \\ Goodwin $^{2,3}$, Sam Pitluck ${ }^{2}$, Konstantinos Liolios ${ }^{2}$, Natalia Ivanova ${ }^{2}$, Konstantinos \\ Mavromatis ${ }^{2}$, Natalia Mikhailova ${ }^{2}$, Amrita Pati ${ }^{2}$, Amy Chen ${ }^{4}$, Krishna Palaniappan ${ }^{4}$, Miriam \\ Land $^{2,5}$, Loren Hauser ${ }^{2,5}$, Yun-Juan Chang ${ }^{2,5}$, Cynthia D. Jeffries ${ }^{2,5}$, Evelyne Brambilla ${ }^{1}$, \\ Manfred Rohde ${ }^{6}$, Markus Göker ${ }^{1}$, John C. Detter ${ }^{2,3}$, Tanja Woyke ${ }^{2}$, James Bristow ${ }^{2}$, Jonathan \\ A. Eisen ${ }^{2,7}$, Victor Markowitz ${ }^{4}$, Philip Hugenholtz ${ }^{2,8}$, Nikos C. Kyrpides ${ }^{2}$, and Hans-Peter \\ Klenk $^{1 *}$ \\ ${ }^{1}$ DSMZ - German Collection of Microorganisms and Cell Cultures GmbH, Braunschweig, \\ Germany \\ ${ }^{2}$ DOE Joint Genome Institute, Walnut Creek, California, USA \\ ${ }^{3}$ Los Alamos National Laboratory, Bioscience Division, Los Alamos, New Mexico, USA \\ ${ }^{4}$ Biological Data Management and Technology Center, Lawrence Berkeley National \\ Laboratory, Berkeley, California, USA \\ ${ }^{5}$ Oak Ridge National Laboratory, Oak Ridge, Tennessee, USA \\ ${ }^{6} \mathrm{HZI}$ - Helmholtz Centre for Infection Research, Braunschweig, Germany \\ ${ }^{7}$ University of California Davis Genome Center, Davis, California, USA \\ ${ }^{8}$ Australian Centre for Ecogenomics, School of Chemistry and Molecular Biosciences, The \\ University of Queensland, Brisbane, Australia \\ *Corresponding author: Hans-Peter Klenk
}

Keywords: strictly anaerobic, nonmotile, Gram-negative, anoxic rice-field soil, mesophilic, chemoorganotrophic, Porphyromonadaceae, GEBA

Paludibacter propionicigenes Ueki et al. 2006 is the type species of the genus Paludibacter, which belongs to the family Porphyromonadaceae. The species is of interest because of the position it occupies in the tree of life where it can be found in close proximity to members of the genus Dysgonomonas. This is the first completed genome sequence of a member of the genus Paludibacter and the third sequence from the family Porphyromonadaceae. The 3,685,504 bp long genome with its 3,054 protein-coding and 64 RNA genes consists of one circular chromosome and is a part of the Genomic Encyclopedia of Bacteria and Archaea project.

\section{Introduction}

Strain WB4 ${ }^{\mathrm{T}}$ (= DSM 17365 = CCUG 53888 = JCM 13257 ) is the type strain of $P$. propionicigenes, which is the type species of the genus Paludibacter $[1,2]$. Currently, there is only one species placed in the genus Paludibacter [1]. The generic name derives from the Latin noun palus -udis meaning swamp or marsh and the Neo-Latin word bacter meaning a rod, referring to a rod living in swamps [2]. The species epithet is derived from the NeoLatin word acidum propionicum meaning propionic acid and the Greek verb gennao meaning to produce, referring to the metabolic property of the species [2]. P. propionicigenes strain $\mathrm{WB}^{\mathrm{T}}$ was isolated together with a number of other strains from rice plant residues in an anoxic rice-field soil in Yamagata, Japan, and described for the first time by Akasaka et al. in 2003 [3]. In 2006 the species was formally described by Ueki et al. and the genus Paludibacter was introduced [2]. No further isolates have been obtained for P. propionicigenes, however, cultivation-independent $16 \mathrm{~S}$ rRNA-dependent molecular investigations showed the presence of $P$. propionicigenes in the rumen of sheep [4]. Here we present a summary classification and a set of features for $P$. propionicigenes 
WB4T, together with the description of the complete genomic sequencing and annotation.

\section{Classification and features}

A representative genomic $16 \mathrm{~S}$ rRNA sequence of strain WB4 ${ }^{\mathrm{T}}$ was compared using NCBI BLAST under default values (e.g., considering only the best 250 hits) with the most recent release of the Greengenes database [5] and the relative frequencies, of taxa and keywords (reduced to their stems [6]) were determined, weighted by BLAST scores. The most frequently occurring genus was Dysgonomonas $(100 \%)$ (8 hits in total). Among all other species, the one yielding the highest score was Dysgonomonas capnocytophagoides, which corresponded to an identity of $91.9 \%$ and a HSP coverage of $83.6 \%$. The highest-scoring environmental sequence was AY212569 ('water $10 \mathrm{~m}$ down- stream manure clone $118 \mathrm{ds} 10$ '), which showed an identity of $99.6 \%$ and a HSP coverage of $100.1 \%$. The five most frequent keywords within the labels of environmental samples which yielded hits were 'digest' (11.7\%), 'anaerob' (6.2\%), 'sludge' (6.1\%), 'wastewater' (6.0\%) and 'mesophile' (5.9\%) (241 hits in total). The single most frequent keyword within the labels of environmental samples which yielded hits of a higher score than the highest scoring species was 'downstream/manure/water' (33.3\%) (1 hit in total).

Figure 1 shows the phylogenetic neighborhood of P. propionicigenes $\mathrm{WB} 4^{\mathrm{T}}$ in a $16 \mathrm{~S}$ rRNA based tree. The three identical 16S rRNA sequences in the genome differ by one nucleotide from the previously published 16S rRNA sequence (AB078842).

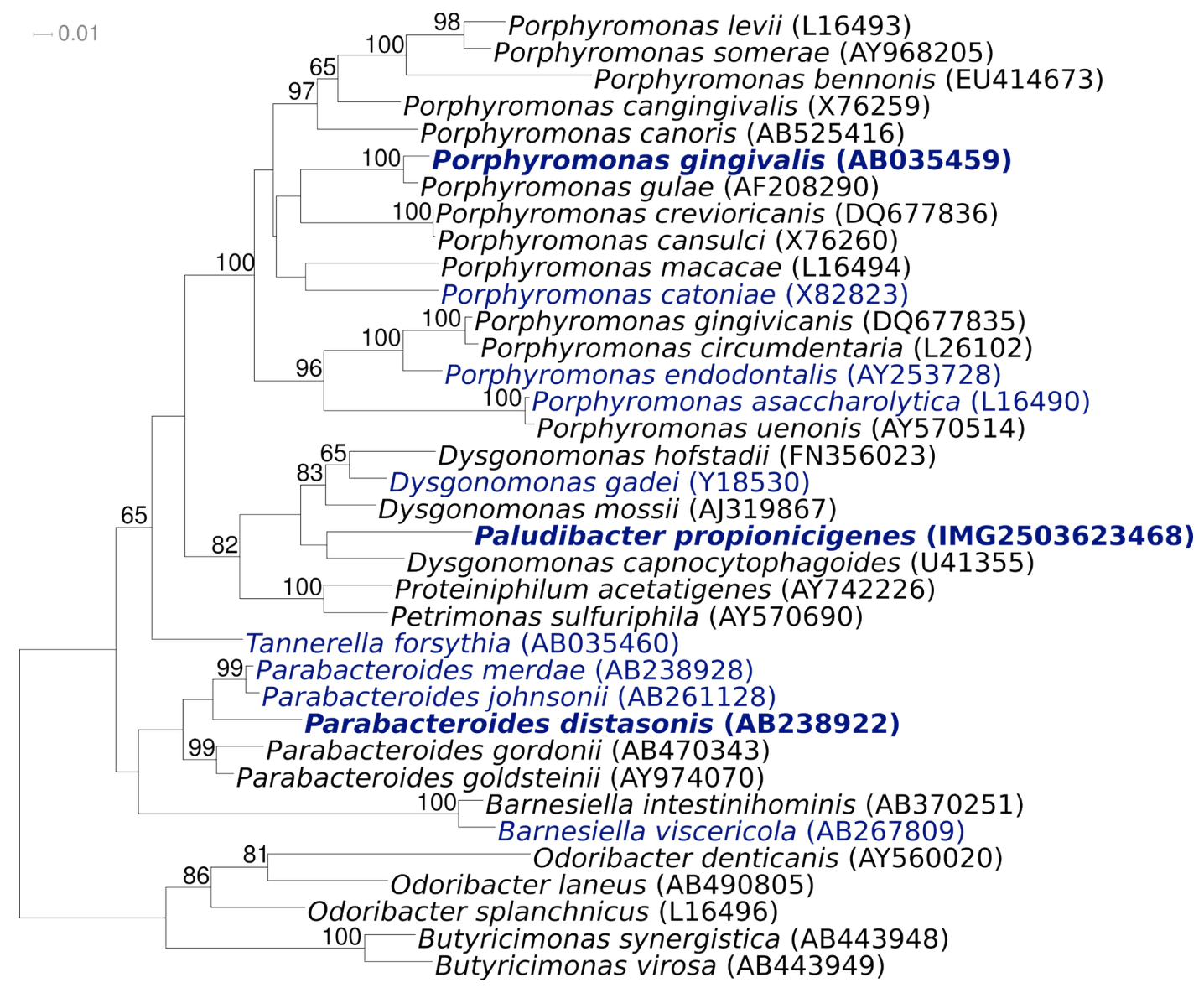

Figure 1. Phylogenetic tree highlighting the position of $P$. propionicigenes relative to the other type strains within the family Porphyromonadaceae. The tree was inferred from 1,400 aligned characters $[7,8]$ of the $16 \mathrm{~S}$ rRNA gene sequence under the maximum likelihood criterion [9] and rooted in accordance with the current taxonomy. The branches are scaled in terms of the expected number of substitutions per site. Numbers above branches are support values from 300 bootstrap replicates [10] if larger than 60\%. Lineages with type strain genome sequencing projects registered in GOLD [11] are shown in blue, published genomes in bold $[12,13]$. 
The cells of $P$. propionicigenes are generally rodshaped $(0.5-0.6 \mu \mathrm{m} \times 1.3-1.7 \mu \mathrm{m})$ with ends that are round or slightly tapered [2]. Elongated cells can also be seen, either as single cells or in short chains (Figure 2). P. propionicigenes is a Gramnegative and non spore-forming bacterium (Table 1). The organism is described to be nonmotile; only eight genes associated with motility were identified in the genome. The organism is strictly anaerobic and chemoorganotrophic [2,3]. The temperature range for growth is between $15^{\circ} \mathrm{C}$ and $35^{\circ} \mathrm{C}$, with an optimum at $30^{\circ} \mathrm{C}$ [2]. The organism does not grow at $37^{\circ} \mathrm{C}$ [2]. The $\mathrm{pH}$ range for growth is 5.0-7.6 with an optimum at $\mathrm{pH} 6.6$ [2]. $\mathrm{NaCl}$ concentrations from $0-0.5 \%(\mathrm{w} / \mathrm{v})$ are tolerated. $P$. propionicigenes is able to utilize arabinose, glucose, fructose, xylose, cellobiose, galactose, mannose, maltose, melibiose, glycogen and soluble starch as growth substrates [2]. The organism does not utilize ribose, lactose, sucrose, melezitose, raffinose, sorbose, rhamnose, trehalose, cellulose, xylan, salicin, dulcitol, inositol, mannitol, sorbitol, ethanol, glycerol, fumarate, ma- late, lactate, succinate or pyruvate [2]. Glucose is fermented to propionate and acetate in a molar ratio of 2:1 as major products and succinate as a minor product [2]. The organism does not reduce nitrate, it does not hydrolyze gelatin or urea and does not produce indole or hydrogen sulfide [2]. $P$. propionicigenes does not grow in the presence of bile salts. Catalase and oxidase are not present in the organism [2].

\section{Chemotaxonomy}

Little chemotaxonomic data are available for strain WB4T. Only the fatty acid composition has been elucidated. The major fatty acids found were anteiso- $\mathrm{C}_{15: 0}(30.8 \%), \mathrm{C}_{15: 0}(19.0 \%)$ and 3-hydroxy anteiso- $\mathrm{C}_{17: 0}(17.9 \%)$ [2]. Also, iso- $\mathrm{C}_{17: 0 \text { 3-он }}(6.2 \%)$ and $\mathrm{C}_{16: 0}(4.9 \%)$ were detected in intermediate amounts whereas iso- $\mathrm{C}_{15: 0}$ 3-OH, iso- $\mathrm{C}_{16: 0} 3-\mathrm{OH}, \mathrm{C}_{15: 0}$ 3он, $\mathrm{C}_{16: 03-\mathrm{OH}}$, iso- $\mathrm{C}_{15: 0}, \mathrm{C}_{14: 0}, \mathrm{C}_{16: 0}$, and $\mathrm{C}_{18: 0}$ were present in minor amounts $(1 \%$ to $5 \%$ of the total fatty acids). Unsaturated fatty acids were not detected [2].

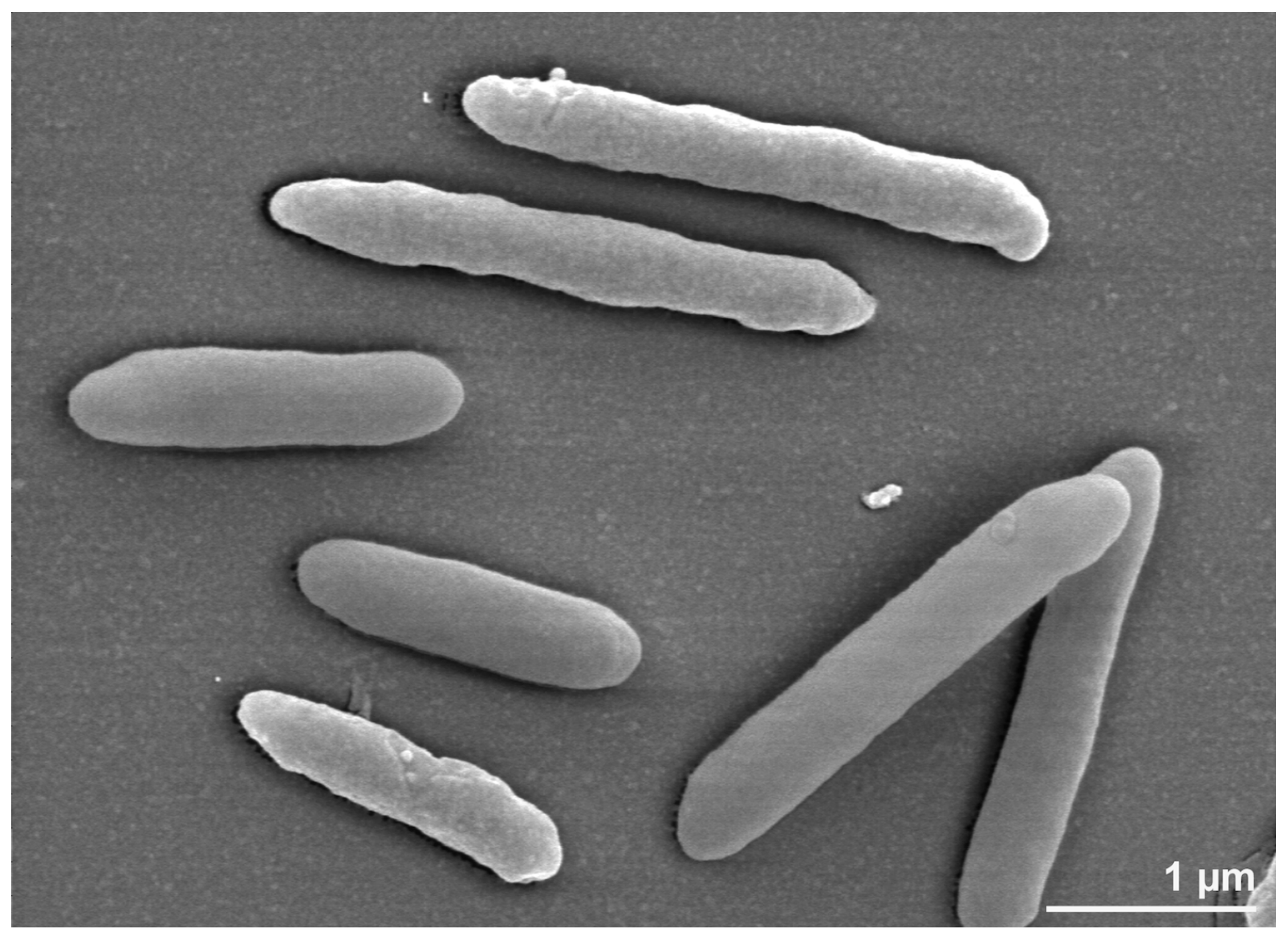

Figure 2. Scanning electron micrograph of $P$. propionicigenes $\mathrm{WB} 4^{\top}$ 
Table 1. Classification and general features of P. propionicigenes WB4 according to the MIGS recommendations [14].

\begin{tabular}{|c|c|c|c|}
\hline MIGS ID & Property & Term & Evidence code \\
\hline & \multirow{8}{*}{ Current classification } & Domain Bacteria & TAS [15] \\
\hline & & Phylum Bacteroidetes & TAS [16] \\
\hline & & Class Bacteroidia & TAS $[16,17]$ \\
\hline & & Order Bacteroidales & TAS [16] \\
\hline & & Family Porphyromonadaceae & TAS [16] \\
\hline & & Genus Paludibacter & TAS [2] \\
\hline & & Species Paludibacter propionicigenes & TAS [2] \\
\hline & & Type strain WB4 & TAS [2] \\
\hline & Gram stain & negative & TAS [3] \\
\hline & Cell shape & rod-shaped & TAS [3] \\
\hline & Motility & non-motile & TAS [2] \\
\hline & Sporulation & none & TAS [3] \\
\hline & Temperature range & $15^{\circ} \mathrm{C}$ to $35^{\circ} \mathrm{C}$ & TAS [3] \\
\hline & Optimum temperature & $30^{\circ} \mathrm{C}$ & TAS [2] \\
\hline & Salinity & normal & NAS \\
\hline \multirow[t]{3}{*}{ MIGS-22 } & Oxygen requirement & strictly anaerobic & TAS [3] \\
\hline & Carbon source & carbohydrates & TAS [3] \\
\hline & Energy source & chemoorganotroph & TAS [3] \\
\hline MIGS-6 & Habitat & soil & TAS [3] \\
\hline MIGS-15 & Biotic relationship & free-living & NAS \\
\hline \multirow[t]{3}{*}{ MIGS-14 } & Pathogenicity & none & NAS \\
\hline & Biosafety level & 1 & TAS [18] \\
\hline & Isolation & rice plant residue in anoxic rice-field soil & TAS [3] \\
\hline MIGS-4 & Geographic location & Yamagata, Japan & TAS [3] \\
\hline MIGS-5 & Sample collection time & 1994 & TAS [3] \\
\hline MIGS-4.1 & Latitude & 38.25 & NAS \\
\hline MIGS-4.2 & Longitude & 140.34 & NAS \\
\hline MIGS-4.3 & Depth & not reported & \\
\hline MIGS-4.4 & Altitude & not reported & \\
\hline
\end{tabular}

Evidence codes - IDA: Inferred from Direct Assay (first time in publication); TAS: Traceable Author Statement (i.e., a direct report exists in the literature); NAS: Non-traceable Author Statement (i.e., not directly observed for the living, isolated sample, but based on a generally accepted property for the species, or anecdotal evidence). These evidence codes are from of the Gene Ontology project [19]. If the evidence code is IDA, then the property was directly observed by one of the authors or an expert mentioned in the acknowledgements.

\section{Genome sequencing and annotation Genome project history}

This organism was selected for sequencing on the basis of its phylogenetic position [20], and is part of the Genomic Encyclopedia of Bacteria and Archaea project [21]. The genome project is deposited in the Genomes OnLine Database [11] and the complete genome sequence is deposited in GenBank. Sequencing, finishing and annotation were performed by the DOE Joint Genome Institute (JGI). A summary of the project information is shown in Table 2. 
Table 2. Genome sequencing project information

\begin{tabular}{lll}
\hline MIGS ID & Property & Term \\
\hline MIGS-31 & Finishing quality & Finished \\
MIGS-28 & Libraries used & Three genomic libraries: one 454 pyrosequence standard library, \\
& one 454 PE library (9 kb insert size), one Illumina library \\
MIGS-29 & Sequencing platforms & Illumina GAii, 454 GS FLX Titanium \\
MIGS-31.2 & Sequencing coverage & $337.6 \times$ Illumina; 28.1 $\times$ pyrosequence \\
MIGS-30 & Assemblers & Newbler version 2 2.3-PreRelease-10-21-2009-gcc-4.1.2- \\
& threads, Velvet, phrap \\
MIGS-32 & Gene calling method & Prodigal 1.4, GenePRIMP \\
& INSDC ID & CP002345 \\
& Genbank Date of Release & December 2, 2010 \\
& GOLD ID & Gc01549 \\
& NCBI project ID & 694427 \\
& Database: IMG-GEBA & 2503538024 \\
SIGS-13 & Source material identifier & DSM 17365 \\
& Project relevance & Tree of Life, GEBA \\
\hline
\end{tabular}

\section{Growth conditions and DNA isolation}

P. propionicigenes WB4T ${ }^{\mathrm{T}}$, DSM 17365, was grown anaerobically in DSMZ medium 104 [22] at $30^{\circ} \mathrm{C}$. DNA was isolated from $0.5-1 \mathrm{~g}$ of cell paste using a MasterPure Gram-positive DNA purification kit (Epicentre MGP04100) following the standard protocol as recommended by the manufacturer, with modification st/DL for cell lysis as described in Wu et al. [21].

\section{Genome sequencing and assembly}

The genome was sequenced using a combination of Illumina and 454 sequencing platforms. All general aspects of library construction and sequencing can be found at the JGI website [23]. Pyrosequencing reads were assembled using the Newbler assembler version 2.3-PreRelease-10-212009-gcc-4.1.2-threads (Roche). The initial Newbler assembly consisting of 26 contigs in one scaffold which was converted into a phrap assembly by [24] making fake reads from the consensus, to collect the read pairs in the 454 paired end library. Illumina GAii sequencing data (967 Mb) was assembled with Velvet [25] and the consensus sequences were shredded into $1.5 \mathrm{~kb}$ overlapped fake reads and assembled together with the 454 data. The 454 draft assembly was based on $93.4 \mathrm{Mb} 454$ draft data and all of the 454 paired end data. Newbler parameters are -consed -a 50 -l 350 -g -m -ml 20. The Phred/Phrap/Consed software package was used for sequence assem- bly and quality assessment in the subsequent finishing process. After the shotgun stage, reads were assembled with parallel phrap (High Performance Software, LLC). Possible mis-assemblies were corrected with gapResolution [23], Dupfinisher, or sequencing cloned bridging PCR fragments with subcloning or transposon bombing (Epicentre Biotechnologies, Madison, WI) [26]. Gaps between contigs were closed by editing in Consed, by PCR and by Bubble PCR primer walks (J.-F.Chang, unpublished). A total of 124 additional reactions and one shatter library were necessary to close the gaps and to raise the quality of the finished sequence. Illumina reads were also used to correct potential base errors and increase consensus quality using a software Polisher developed at JGI [27]. The error rate of the completed genome sequence is less than 1 in 100,000 . Together, the combination of the Illumina and 454 sequencing platforms provided $365.7 \times$ coverage of the genome. The final assembly contained 333,397 pyrosequence and 34,564,373 Illumina reads.

\section{Genome annotation}

Genes were identified using Prodigal [28] as part of the Oak Ridge National Laboratory genome annotation pipeline, followed by a round of manual curation using the JGI GenePRIMP pipeline [29]. The predicted CDSs were translated and used to search the National Center for Biotechnology In- 
formation (NCBI) nonredundant database, UniProt, TIGR-Fam, Pfam, PRIAM, KEGG, COG, and InterPro databases. Additional gene prediction analysis and functional annotation was performed within the Integrated Microbial Genomes - Expert Review (IMG-ER) platform [30].

\section{Genome properties}

The genome consists of a 3,685,504 bp long chromosome with a GC content of $38.9 \%$ (Figure 3 and
Table 3). Of the 3,118 genes predicted, 3,054 were protein-coding genes, and 64 RNAs; 34 pseudogenes were also identified. The majority of the protein-coding genes (65.8\%) were assigned with a putative function while the remaining ones were annotated as hypothetical proteins. The distribution of genes into COGs functional categories is presented in Table 4.

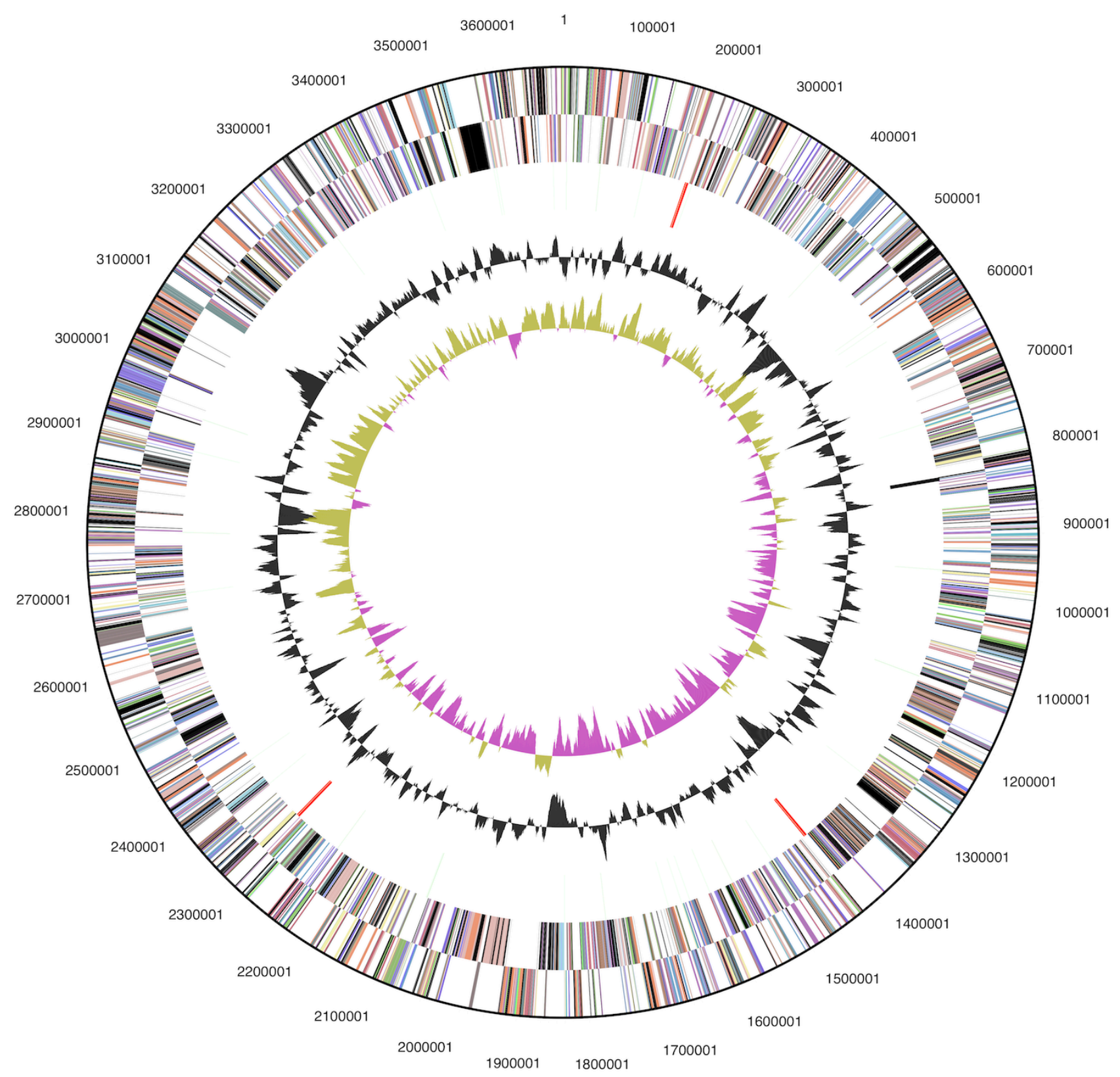

Figure 3. Graphical circular map of the chromosome. From outside to the center: Genes on forward strand (color by COG categories), Genes on reverse strand (color by COG categories), RNA genes (tRNAs green, rRNAs red, other RNAs black), GC content, GC skew. 
Table 3. Genome Statistics

\begin{tabular}{lrr}
\hline Attribute & Value & \% of Total \\
\hline Genome size (bp) & $3,685,504$ & $100.00 \%$ \\
DNA coding region (bp) & $3,225,817$ & $87.53 \%$ \\
DNA G+C content (bp) & $1,432,064$ & $38.86 \%$ \\
Number of replicons & 1 & \\
Extrachromosomal elements & 0 & \\
Total genes & 3,118 & $100.00 \%$ \\
RNA genes & 64 & $2.05 \%$ \\
rRNA operons & 3 & \\
Protein-coding genes & 3,054 & $97.95 \%$ \\
Pseudo genes & 34 & $1.09 \%$ \\
Genes with function prediction & 2,051 & $65.78 \%$ \\
Genes in paralog clusters & 325 & $10.42 \%$ \\
Genes assigned to COGs & 2,005 & $64.30 \%$ \\
Genes assigned Pfam domains & 2,205 & $70.72 \%$ \\
Genes with signal peptides & 843 & $27.04 \%$ \\
Genes with transmembrane helices & 784 & $25.14 \%$ \\
CRISPR repeats & 2 & \\
\hline
\end{tabular}

Table 4. Number of genes associated with the general COG functional categories

\begin{tabular}{lrrl}
\hline Code & value & \%age & Description \\
\hline J & 149 & 6.8 & Translation, ribosomal structure and biogenesis \\
A & 0 & 0 & RNA processing and modification \\
K & 136 & 6.2 & Transcription \\
L & 101 & 4.6 & Replication, recombination and repair \\
B & 0 & 0 & Chromatin structure and dynamics \\
D & 22 & 1.0 & Cell cycle control, cell division, chromosome partitioning \\
Y & 0 & 0 & Nuclear structure \\
V & 48 & 2.2 & Defense mechanisms \\
T & 99 & 4.5 & Signal transduction mechanisms \\
M & 232 & 10.6 & Cell wall/membrane/envelope biogenesis \\
N & 8 & 0.4 & Cell motility \\
Z & 0 & 0 & Cytoskeleton \\
W & 0 & 0 & Extracellular structures \\
U & 40 & 1.8 & Intracellular trafficking, secretion, and vesicular transport \\
O & 80 & 3.7 & Posttranslational modification, protein turnover, chaperones \\
C & 108 & 5.0 & Energy production and conversion \\
G & 172 & 7.9 & Carbohydrate transport and metabolism \\
E & 166 & 7.6 & Amino acid transport and metabolism \\
F & 61 & 2.8 & Nucleotide transport and metabolism \\
H & 128 & 5.9 & Coenzyme transport and metabolism \\
I & 67 & 3.1 & Lipid transport and metabolism \\
P & 131 & 6.0 & Inorganic ion transport and metabolism \\
Q & 24 & 1.1 & Secondary metabolites biosynthesis, transport and catabolism \\
R & 256 & 11.7 & General function prediction only \\
S & 153 & 7.0 & Function unknown \\
- & 1,113 & 35.7 & Not in COGs \\
\hline
\end{tabular}




\section{Acknowledgements}

We would like to gratefully acknowledge the help of Sabine Welnitz (DSMZ) for growing P. propionicigenes cultures. This work was performed under the auspices of the US Department of Energy Office of Science, Biological and Environmental Research Program, and by the University of California, Lawrence Berkeley National Laboratory under contract No. DE-AC02-05CH11231,

\section{References}

1. Garrity G. NamesforLife. BrowserTool takes expertise out of the database and puts it right in the browser. Microbiol Today 2010; 7:1.

2. Ueki A, Akasaka H, Suzuki D, Ueki K. Paludibacter propionicigenes gen. nov., sp. nov., a novel strictly anaerobic, Gram-negative, propionateproducing bacterium isolated from plant residue in irrigated rice-field soil in Japan. Int J Syst Evol Microbiol 2006; 56:39-44. PubMed doi:10.1099/ijs.0.63896-0

3. Akasaka H, Izawa T, Ueki K, Ueki A. Phylogeny of numerically abundant culturable anaerobic bacteria associated with degradation of rice plant residue in Japanese paddy field soil. FEMS Microbiol Ecol 2003; 43:149-161. PubMed doi:10.1111/j.1574-6941.2003.tb01054.x

4. Pei CX, Liu Q, Dong CS, Li H, Jiang JB, Gao WJ. Diversity and abundance of the bacterial $16 \mathrm{~S}$ rRNA gene sequences in forestomach of alpacas (Lama pacos) and sheep (Ovis aries). Anaerobe 2010; 16:426-432. PubMed doi:10.1016/j.anaerobe.2010.06.004

5. DeSantis TZ, Hugenholtz P, Larsen N, Rojas M, Brodie EL, Keller K, Huber T, Dalevi D, Hu P, Andersen GL. Greengenes, a Chimera-Checked 16S rRNA Gene Database and Workbench Compatible with ARB. Appl Environ Microbiol 2006; 72:5069-5072. PubMed doi:10.1128/AEM.03006-05

6. Porter MF. An algorithm for suffix stripping. Program: electronic library and information systems 1980; 14:130-137.

7. Lee C, Grasso C, Sharlow MF. Multiple sequence alignment using partial order graphs. Bioinformatics 2002; 18:452-464. PubMed doi:10.1093/bioinformatics/18.3.452

8. Castresana J. Selection of conserved blocks from multiple alignments for their use in phylogenetic analysis. Mol Biol Evol 2000; 17:540-552. PubMed

9. Stamatakis A, Hoover P, Rougemont J. A rapid bootstrap algorithm for the RAxML web-servers.
Lawrence Livermore National Laboratory under Contract No. DE-AC52-07NA27344, and Los Alamos National Laboratory under contract No. DE-AC0206NA25396, UT-Battelle and Oak Ridge National Laboratory under contract DE-AC05-000R22725, as well as German Research Foundation (DFG) INST 599/1-2.

Syst Biol 2008; 57:758-771. PubMed doi:10.1080/10635150802429642

10. Pattengale ND, Alipour M, Bininda-Emonds ORP, Moret BME, Stamatakis A. How Many Bootstrap Replicates Are Necessary? Lect Notes Comput Sci 2009; 5541:184-200. doi:10.1007/978-3-642$\underline{02008-7 \quad 13}$

11. Liolios K, Chen IM, Mavromatis K, Tavernarakis $\mathrm{N}$, Hugenholtz P, Markowitz VM, Kyrpides NC. The Genomes OnLine Database (GOLD) in 2009: status of genomic and metagenomic projects and their associated metadata. Nucleic Acids Res 2010; 38:D346-D354. PubMed doi:10.1093/nar/gkp848

12. Xu J, Mahowald MA, Ley RE, Lozupone CA, Hamady M, Martens EC, Henrissat B, Coutinho PM, Minx $\mathrm{P}$, Latreille $\mathrm{P}$, et al. Evolution of symbiotic bacteria in the distal human intestine. PLoS Biol 2007; 5:e156. PubMed doi:10.1371/journal.pbio.0050156

13. Naito $M$, Hirakawa $H$, Yamashita $A$, Ohara $N$, Shoji M, Yukitake H, Nakayama K, Toh H, Yoshimura F, Kuhara S, et al. Determination of the genome sequence of Porphyromonas gingivalis strain ATCC 33277 and genomic comparison with strain W83 revealed extensive genome rearrangements in $P$. gingivalis. DNA Res 2008; 15:215-225. PubMed doi:10.1093/dnares/dsn013

14. Field D, Garrity G, Gray T, Morrison N, Selengut J, Sterk P, Tatusova T, Thomson N, Allen MJ, Angiuoli SV, et al. The minimum information about a genome sequence (MIGS) specification. Nat Biotechnol 2008; 26:541-547. PubMed doi:10.1038/nbt1360

15. Woese CR, Kandler O, Wheelis ML. Towards a natural system of organisms: proposal for the domains Archaea, Bacteria, and Eucarya. Proc Natl Acad Sci USA 1990; 87:4576-4579. PubMed doi:10.1073/pnas.87.12.4576

16. Garrity GM, Holt JG. Taxonomic Outline of the Archaea and Bacteria. In: Garrity GM, Boone DR, Castenholz RW (eds), Bergey's Manual of Syste- 
matic Bacteriology, Second Edition, Volume 1, Springer, New York, 2001, p. 155-166.

17. Ludwig W, Euzeby J, Whitman WG. Draft taxonomic outline of the Bacteroidetes, Planctomycetes, Chlamydiae, Spirochaetes, Fibrobacteres, Fusobacteria, Acidobacteria, Verrucomicrobia, Dictyoglomi, and Gemmatimonadetes. http://www.bergeys.org/outlines/Bergeys_Vol_4 Outline.pdf.

18. BAuA. Classification of bacteria and archaea in risk groups. TRBA 2005; 466:84.

19. Ashburner M, Ball CA, Blake JA, Botstein D, Butler $\mathrm{H}$, Cherry JM, Davis AP, Dolinski K, Dwight SS, Eppig JT, et al. Gene Ontology: tool for the unification of biology. Nat Genet 2000; 25:25-29. $\underline{\text { PubMed doi: } 10.1038 / 75556}$

20. Klenk HP, Goeker M. En route to a genome-based classification of Archaea and Bacteria? Syst Appl Microbiol 2010; 33:175-182. PubMed doi:10.1016/j.syapm.2010.03.003

21. Wu D, Hugenholtz P, Mavromatis K, Pukall R, Dalin E, Ivanova NN, Kunin V, Goodwin L, Wu $M$, Tindall BJ, et al. A phylogeny-driven genomic encyclopaedia of Bacteria and Archaea. Nature 2009; 462:1056-1060. $\underline{\text { PubMed }}$ doi:10.1038/nature08656

22. List of growth media used at DSMZ: http://www.dsmz.de/microorganisms/media_list.p hp.

23. DOE Joint Genome Institute. http://www.jgi.doe.gov
24. Phrap and Phred for Windows. MacOS, Linux, and Unix. http://www.phrap.com

25. Zerbino DR, Birney E. Velvet: algorithms for de novo short read assembly using de Bruijn graphs. Genome Res 2008; 18:821-829. PubMed doi:10.1101/gr.074492.107

26. Han C, Chain P. 2006. Finishing repeat regions automatically with Dupfinisher. in Proceeding of the 2006 international conference on bioinformatics \& computational biology. Edited by Hamid R. Arabnia \& Homayoun Valafar, CSREA Press. June 26- 29, 2006: 141-146.30.

27. Lapidus A, LaButti K, Foster B, Lowry S, Trong S, Goltsman E. POLISHER: An effective tool for using ultra short reads in microbial genome assembly and finishing. AGBT, Marco Island, FL, 2008.

28. Hyatt D, Chen GL, LoCascio PF, Land ML, Larimer FW, Hauser LJ. Prodigal: prokaryotic gene recognition and translation initiation site identification. BMC Bioinformatics 2010; 11:119. PubMed doi:10.1186/1471-2105-11-119

29. Pati A, Ivanova NN, Mikhailova N, Ovchinnikova G, Hooper SD, Lykidis A, Kyrpides NC. GenePRIMP: a gene prediction improvement pipeline for prokaryotic genomes. Nat Methods 2010; 7:455-457. PubMed doi:10.1038/nmeth.1457

30. Markowitz VM, Ivanova NN, Chen IMA, Chu K, Kyrpides NC. IMG ER: a system for microbial genome annotation expert review and curation. Bioinformatics 2009; 25:2271-2278. PubMed doi:10.1093/bioinformatics/btp393 